\title{
Demokratie und Öffentlichkeit
}

\author{
Eisenegger, Mark ; Udris, Linards
}

\begin{abstract}
Im 5. Jahrhundert v. Chr. entstand im antiken Stadtstaat Athen eine der wirkmächtigsten Utopien der Menschheit: Die Idee, dass eine freie öffentliche Kommunikation Vernunft (Logos) und die legitimste aller Gesellschaftsformen sichert - die Demokratie (vgl. Arendt, 1992; Imhof, 1998). Nur das, was sich im öffentlichen Abwägen unterschiedlicher Standpunkte durchsetzen kann, so waren die alten Griechen überzeugt, kann mit der Zustimmung aller rechnen.Die Verwirklichung dieses Ideals war an klare Bedingungen geknüpft. Es musste mit der Agora, dem Marktplatz der Ideen, einen gemeinsamen Ort der öffentlichen Debatte geben. Die Kommunikation auf der Agora sollte so gestaltet ein, dass sich die "sanfte Gewalt des besseren Arguments" bestmöglich zur Geltung bringen kann. Wie präsentiert sich die gegenwärtige, realexistierende Öffentlichkeit im Licht des aufklärerischen Ideals? Die moderne, mediale Agora wankt gewaltig. Ein wichtiger Faktor ist die Digitalisierung der öffentlichen Kommunikation, und diese wird wiederum immer stärker von globalen Tech-Giganten wie Google und Facebook bestimmt. Insgesamt stellt die >Plattformisierung< - d. h. der Bedeutungsgewinn von Facebook, Google und Co. - die mediale Agora vor grosse Probleme. In diesem Beitrag skizzieren wir dies anhand von drei bewusst zugespitzten Thesen. Ausleitend diskutieren wir, welche Gegenkräfte in der Schweiz (noch) wirken und was wir tun können, um die Öffentlichkeit und damit auch die Demokratie wieder zu stärken.
\end{abstract}

Posted at the Zurich Open Repository and Archive, University of Zurich

ZORA URL: https://doi.org/10.5167/uzh-175840

Book Section

Published Version

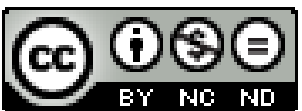

The following work is licensed under a Creative Commons: Attribution-NonCommercial-NoDerivatives 4.0 International (CC BY-NC-ND 4.0) License.

Originally published at:

Eisenegger, Mark; Udris, Linards (2019). Demokratie und Öffentlichkeit. In: Daellenbach, Ruth; Ringger, Beat; Zwicky, Pascal. Reclaim democracy : die Demokratie stärken und weiterentwickeln. Zürich: Edition 8, 73-81. 


\title{
Demokratie und Öffentlichkeit
}

\author{
Mark Eisenegger, Linards Udris
}

Im 5. Jahrhundert v. Chr. entstand im antiken Stadtstaat Athen eine der wirkmächtigsten Utopien der Menschheit: Die Idee, dass eine freie öffentliche Kommunikation Vernunft (Logos) und die legitimste aller Gesellschaftsformen sichert - die Demokratie (vgl. Arendt, 1992; Imhof, 1998). Nur das, was sich im öffentlichen Abwägen unterschiedlicher Standpunkte durchsetzen kann, so waren die alten Griechen überzeugt, kann mit der Zustimmung aller rechnen.

Die Verwirklichung dieses Ideals war an klare Bedingungen geknüpft. Es musste mit der Agora, dem Marktplatz der Ideen, einen gemeinsamen Ort der öffentlichen Debatte geben. Die Kommunikation auf der Agora sollte so gestaltet sein, dass sich die "sanfte Gewalt des besseren Arguments" bestmöglich zur Geltung bringen kann.

Nicht Status oder Macht sollten den Diskurs beherrschen, sondern die Überzeugungskraft der Argumente. Argumente sollten gegen Argumente getauscht werden und nicht gegen Personen gerichtet sein. Menschen sollten aus unterschiedlichen Kontexten zusammenkommen und die Bereitschaft mitbringen, die eigene Position gegebenenfalls zu revidieren. Nur so könne sich auf der Agora Schwarmintelligenz einstellen.

Das antike Ideal wurde zum Kern der Aufklärungsbewegung im 18. Jahrhundert. Ohne sie gäbe es keine modernen, demokratischen Gesellschaften heutiger Prägung. Indem sich sukzessive eine massenmediale Agora formierte, wurde die Utopie der demokratischen Gesellschaft auf grossflächige Gebiete wie den Nationalstaat übertragbar. Der Aufklärungsbewegung verdanken wir den modernen Rechtsstaat, die Bürger- und Menschenrechte sowie die Einsicht, dass die freie öffentliche Kommunikation die höchste Instanz in der Gesellschaft ist, weil sich vor ihr alle rechtfertigen müssen, auch die Mächtigen der Gesellschaft. Die in Demokratien verankerte Pressefreiheit, die Ausgestaltung von öffentlich finanzierten Medien oder die Journalistenpreise, mit denen ein kritischer, substanzieller und einordnender Journalismus belohnt wird, zeugen davon, dass diese Normen nach wie vor wirkmächtig sind. Die Aufklärung ist immer noch die zentrale Leitlinie, an der sich (westliche) demokratische Gesellschaften orientieren. Empirisch gesehen, hat die Öffentlichkeit ein solches Ideal nie vollständig erreicht. Immer wieder wurden gerade die etablierten Medien als zentrale Pfeiler der Öffentlichkeit vom Staat und/oder von der Wirt- 
schaft für ihre Zwecke instrumentalisiert. Auch in der viel zitierten, von Jürgen Habermas beschriebenen Öffentlichkeit an der Schwelle zwischen Feudalismus und Demokratie Ende des 19. Jahrhunderts waren zudem wichtige soziale Gruppen wie Frauen und Arme ausgeschlossen. Doch die Diskurse der Aufklärung mit ihrem universalistischen Charakter wiesen und weisen stets auch ein Potenzial für mehr Inklusion und Partizipation auf (vgl. dazu Wessler, 2018). Und gerade auch die beständige Öffentlichkeits-Kritik in Demokratien bekräftigt immer wieder aufs Neue die antike und aufklärerische Utopie, was öffentliche Kommunikation für die Demokratie leisten soll.

Wie präsentiert sich die gegenwärtige, realexistierende Öffentlichkeit im Licht des aufklärerischen Ideals? Die moderne, mediale Agora wankt gewaltig. Ein wichtiger Faktor ist die Digitalisierung der öffentlichen Kommunikation, und diese wird wiederum immer stärker von globalen Tech-Giganten wie Google und Facebook bestimmt. Insgesamt stellt die >Plattformisierung - d.h. der Bedeutungsgewinn von Facebook, Google und Co. - die mediale Agora vor grosse Probleme. In diesem Beitrag skizzieren wir dies anhand von drei bewusst zugespitzten Thesen. Ausleitend diskutieren wir, welche Gegenkräfte in der Schweiz (noch) wirken und was wir tun können, um die Öffentlichkeit und damit auch die Demokratie wieder zu stärken.

\section{Immer mehr Menschen bleiben der medialen Agora fern}

Im Zuge des digitalen Strukturwandels der Öffentlichkeit (vgl. Eisenegger, 2017) findet nicht nur eine Verschiebung der Newsnutzung auf Onlinekanäle statt: Immer mehr Nutzer wenden sich auch grundsätzlich vom Informationsjournalismus ab. Das können wir zeigen auf der Grundlage von repräsentativen Befragungen, die wir seit 2009 durchführen und die wir unter anderem in unserem `Jahrbuch Qualität der Medien ‘ festhalten (vgl. fög, 2018; Schneider \& Eisenegger, 2016, 2018). Seit 2009 nimmt nämlich die Gruppe der sogenannten >News-Deprivierten` stetig und signifikant zu. News-Deprivierte sind Nutzerinnen und Nutzer, die News weit unterdurchschnittlich konsumieren. Wenn News-Deprivierte überhaupt Nachrichten nutzen, dann vorab aus qualitätsschwachen Informationsmedien und vor allem via Social Media. Bedenklich ist: Die Gruppe der NewsDeprivierten ist von allen erfassten Nutzergruppen in den letzten zehn Jahren am stärksten gewachsen. Allein von 2017 auf 2018 haben die News-Deprivierten nochmals um 5 Prozentpunkte zugelegt und sind mit 36\% die heute mit Abstand grösste Nutzungsgruppe in der Schweiz. Zoomt man auf die Altersgruppe der 16- bis 29-Jährigen ein, so sind mehr als die Hälfte, nämlich 53\% der jungen Erwachsenen, den News-Deprivierten zuzurechnen. 
Wir haben bewusst den Begriff >News-Deprivation` und nicht >NewsVerweigerung` oder `News-Abstinenz` gewählt. Denn es handelt sich bei diesem unterdurchschnittlichen Newskonsum nicht unbedingt um das Resultat aktiver Verweigerung.

Ausschlaggebend sind vielmehr zeitliche Verdrängungseffekte, die sich auch durch das wachsende (Unterhaltungs-)Angebot ergeben. Denn die News-Deprivierten sind durchaus medienaffin und investieren viel Zeit in den Medienkonsum, aber eben nicht zu Newszwecken. Stattdessen dominieren andere Medienaktivitäten, etwa das Socializing, d.h. der Austausch bzw. das Chatten mit Bekannten und `friends` oder die Nutzung von Streamingdiensten wie Spotify oder Netflix. Empirisches Anschauungsmaterial liefert das Medienkonsumverhalten unterwegs in der S-Bahn: Nicht News, sondern die letzte Folge der eigenen US-Lieblingsserie füllen immer häufiger die kleinen Bildschirme der Smartphones. Die meiste Zeit wird heute in der Schweiz denn auch tatsächlich mit Angeboten global tätiger Unternehmen wie Google, Facebook, Youtube, Instagram, WhatsApp, Spotify oder Netflix verbracht. Viermal mehr Nutzungszeit wird für diese Angebote investiert als für die fünf am häufigsten genutzten Internetseiten der Schweizer Medien zusammen (vgl. Schweizerische Eidgenossenschaft, 2018, 10).

Wenn sich immer mehr Leute nur noch selten auf der medialen Agora aufhalten oder ihr ganz fernbleiben, wirkt sich dies auf deren Wahrnehmung der Gesellschaft aus. In den Befragungen haben wir die Mediennutzenden danach gefragt, welche Themen sie am meisten wahrnehmen. Die Untersuchung zeigt: >News-Deprivierte`schenken vor allem Softnews oder `bedrohlichen $>$ und emotional aufgeladenen Ereignissen wie Terrorismus oder 〉Flüchtlingskrise`Aufmerksamkeit (vgl. Schneider \& Eisenegger, 2016). Dies macht sie potenziell anfällig für populistische Angstpolitik mit ihren scheinbar einfachen Lösungen.

\section{Die Digitalisierung der Agora fördert die Unvernunft}

Viele Forscherinnen und Forscher, Journalistinnen und Journalisten haben lange geglaubt, dass die Digitalisierung der medialen Agora der Aufklärung zu einem neuen Siegeszug verhelfen würde. Die Hoffnung war, dass im Internet das rationale, herrschaftsemanzipierte Räsonnement gefördert und die Demokratie gestärkt würde. Tatsächlich sind auch neue, zivilgesellschaftlich rückgebundene Online-Medien von hoher Qualität wie beispielsweise Mediapart in Frankreich entstanden.

Der Grundbefund jedoch, dass solche neuen Online-Medien vielmehr die Ausnahme als die Regel sind und dass es sich bei den wesentlich reichweitenstärkeren sozialen Medien von ihrer Grundtendenz her um Emotionsmedien handelt, lässt uns an der Hoffnung auf mehr Räsonnement im 
Internet zweifeln. Tatsächlich provozieren emotional aufgeladene Beiträge mehr Reaktionen auf sozialen Medien als nicht emotionale (vgl. Lucht \& Udris \& Vogler, 2018) und sie werden durch Algorithmen auch prominent angezeigt (vgl. Stieglitz \& Dang-Xuan, 2012; Stieglitz \& Dang-Xuan, 2013). Die Bevorzugung emotionaler Inhalte bildet die Voraussetzung für den Erfolg des Geschäftsmodells der sozialen Plattformen, das mittels niederschwelliger Klicks, Likes, Shares oder anderer Reaktionen laufend ökonomisch verwertbare Nutzerdaten akkumulieren will. Nicht das rationale Abwägen von Argumenten, sondern schrille, laute Töne fallen auf sozialen Medien auf einen günstigen Nährboden. Das zeigt sich auch an der Zunahme von inzivilen Online-Nutzerkommentaren, in denen basale Spielregeln des respektvollen Austauschs nicht eingehalten werden und die gerade deshalb in sozialen Netzwerken besonders hohe Nutzerreaktionen auslösen.

Ganz generell verändern die sozialen Netzwerke somit, ob und wie wir miteinander interagieren und argumentieren. Erst seit relativ kurzer Zeit wird sehr viel Forschung betrieben zu `Filterblasen $<$ und $`$ Echokammern $<-$ mit unterschiedlichen Resultaten und noch zu wenig gesicherten Erkenntnissen. Manche beobachten eine klare Fragmentierung der Öffentlichkeit, also ein Aufsplittern in abgeschottete Kleinst-Agoren, in denen Gleichgesinnte unter sich bleiben. In dieser Nestwärme unter >friends ‘ finden auch noch die abstrusesten Ideen Bestätigung. Andere aber halten dagegen, dass Menschen unter anderem auch auf Facebook durchaus mit verschiedenen Quellen konfrontiert werden. Auch wir können keine abschliessende Antwort geben, doch wir halten folgende Punkte für besonders wichtig:

Tatsächlich gibt es nach wie vor auch auf den sozialen Netzwerken reichweitenstarke Medien, die von vielen Menschen genutzt werden. SRF, 20 Minuten oder - in geringerem Masse - die NZZ erreichen hohe Resonanz über verschiedene Bevölkerungsgruppen hinweg. Doch der Punkt ist, dass auf Social Media das News-Angebot derselben Medien höchst unterschiedlich genutzt wird. Während beispielsweise die Community der deutschen AfD-nahen Rechten primär kritische NZZ-Beiträge über die Flüchtlingspolitik von Angela Merkel teilt, konsumiert und teilt die Schweizer Twitter-Community völlig andere Inhalte der NZZ (vgl. Rauchfleisch \& Vogler \& Eisenegger, 2017). Die Nutzer leben also teilweise auf völlig unterschiedlichen Informationsinseln, und zwar selbst dann, wenn sie die gleichen Medien nutzen.

Dazu kommt, dass die Aneignung der Medien bei den verschiedenen Communities eine ganz andere ist. Anders als auf den ersten Blick vermutet, nutzen beispielsweise auch die vermeintlich ‘abgehängten` Personen im rechtspopulistischen Milieu in Deutschland den öffentlichen Rundfunk und nicht bloss >Alternativmedien aus den Weiten des Internets. Aber sie nutzen den öffentlichen Rundfunk aus völlig anderen Motiven, nämlich als 
Angriffsfläche ihrer Ablehnung der so genannten `Mainstream-Medien (vgl. Schulz, 2018).

Gesamthaft reduziert die Plattformisierung und die damit verbundene Zunahme des Medienkonsums über Facebook, Google und Co. die Integrationsfunktion medialer Kommunikation. Zunehmend mehr Nutzerinnen und Nutzer bleiben der Agora ganz fern oder nehmen nur einen Bruchteil der Debatte wahr, nämlich jenen, der ins eigene Weltbild passt oder der bei der eigenen Community auf Widerhall stösst. Anstatt konträre Standpunkte abzuwägen und dadurch der Vernunft zum Durchbruch zu verhelfen, schaukelt man sich in `Echokammern Drastik ersetzt Präzision; Wut verdrängt Geist. Eine Gesellschaft aber, die im kommunikativen Modus der Selbstbestätigung operiert, wird dümmer.

\section{Die mediale Agora wankt und ist unter Beschuss}

Die Plattformisierung schwächt die mediale Agora ökonomisch und politisch. Das System der Schweizer Informationsmedien kommt in ökonomischer Hinsicht gleich mehrfach unter Druck. Momentan existiert kein nachhaltiges Geschäftsmodell für Informationspublizistik auf den digitalen Kanälen. Das liegt einerseits an der äusserst tiefen Zahlungsbereitschaft für News - nur 12\% der Schweizerinnen und Schweizer sind noch bereit, für Onlinenews Geld auszugeben. Dazu gehört, dass der Medienkonsum immer weniger direkt über `Medienmarken respektive Medientitel erfolgt, sondern dass Nutzer in ihrem semergenten Medienkonsum ein personalisiertes Bouquet aus Medienbeiträgen erhalten. Während beim klassischen Konsum das Angebot beispielsweise von einer bestimmten Zeitung vorgegeben wird, entsteht beim >emergenten $<$ Konsum dieses Angebot erst durch die Neu-Bündelung und dynamische Aggregierung von Inhalten aus unterschiedlichsten Quellen im persönlichen Feed oder in den Trefferlisten der Nutzerin oder des Nutzers. Annähernd ein Viertel der Schweizerinnen und Schweizer konsumieren News bereits vorwiegend emergent über soziale Plattformen und Newsaggregatoren oder indem sie Stichworte in Suchmaschinen eingeben. Für die traditionellen Informationsmedienanbieter ist diese Entwicklung problematisch. Emergente Mediennutzende bringen News nicht mit den wirklichen Produzenten der Inhalte in Verbindung, sondern mit den grossen Plattformen. Sie wissen noch, dass sie etwas rauf Facebook ' gelesen haben, aber nicht mehr, von wem die Nachricht stammt (vgl. Kalogeropoulos \& Newman, 2017). Unter diesem Trend zu »Homeless media" (Marconi 2015) leidet die Markenbindung an traditionelle Newsanbieter. Und dies belastet die ohnehin tiefe Zahlungsbereitschaft für Onlinenews zusätzlich.

Ökonomisch schwierig ist auch die Situation im Onlinewerbemarkt. Die Plattformisierung, d.h. die Dominanz der globalen Tech-Intermediä- 
ren, entzieht dem Schweizer Mediensystem Werbeeinkünfte in substanziellem Ausmass. Die Einnahmen aus Displaywerbung, der primären Onlinewerbeform für Informationspublizistik, betragen total gegenwärtig nur 265 Millionen Franken. Zwei Drittel (67\%) der Online-Werbeerträge, also rund 1,4 Milliarden Franken, stammen aus Suchmaschinenwerbung und fliessen vor allem zu Google ab (vgl. Stiftung Werbestatistik Schweiz, 2018). $\mathrm{Zu}$ den Erträgen von Social-Media-Anbietern existieren für die Schweiz bislang nur Schätzungen. Diese gehen für Facebook von jährlichen Einnahmen um 210 Millionen Franken aus (vgl. von Matt, 2018). Parallel zur wachsenden Dominanz der Tech-Intermediäre im Onlinewerbemarkt schwinden die Einnahmen aus dem Werbegeschäft mit den gedruckten Zeitungen. Sie reduzieren sich in den letzten zehn Jahren um 1,4 Milliarden Franken und betragen aktuell mit 1,1 Milliarden Franken nicht einmal mehr die Hälfte des ursprünglichen Volumens. Das bedeutet, dass im Jahr 2017 in der Schweiz Google alleine mehr im Werbemarkt verdiente als die gesamte Presse zusammen (vgl. Stiftung Werbestatistik Schweiz, 2018). Die Entwicklungen in der Schweiz decken sich mit internationalen Befunden. Auch in den USA wandert der Grossteil (63\%) der Online-Werbeeinnahmen auf das Konto von Google und Facebook (vgl. eMarketer, 2017).

Auch politisch kommt die mediale Agora unter Druck. Ausgerechnet jene Medien, die sich am meisten an Qualitätsstandards und am Prinzip der »sanften Gewalt des besseren Arguments« orientieren, werden frontal angegriffen. Das gilt zunächst für die öffentlichen Medien. In mehreren europäischen Ländern geraten öffentliche Medienhäuser unter Beschuss: sowohl von Verlagshäusern als auch von rechtspopulistischen Parteien. Private Medienorganisationen stilisieren den öffentlichen Rundfunk mitunter zum Hauptproblem der aktuellen Finanzierungskrise der privaten Medien - dies unter Ausblendung der wahren Ursachen wie der massiv gewachsenen Konkurrenz der globalen Tech-Giganten oder der durch die privaten Medien mitverschuldeten Gratiskultur. Rechtspopulistische Parteien kritisieren die angebliche "Staatsnähe " und die angebliche "Linkslastigkeit« des öffentlichen Rundfunks.

Zwar haben sich die Schweizer Stimmberechtigten im März 2018 mit grosser Mehrheit gegen die Abschaffung der Rundfunkgebühren ausgesprochen (`No Billag‘-Initiative). Dass die Initiative einen öffentlichen Rundfunk, der in allen Landesteilen ein substanzielles und attraktives Programm anbietet, in Zukunft verunmöglicht hätte, erschien vielen $\mathrm{zu}$ radikal. Dennoch bedeutet die starke Ablehnung von `No Billag « keine enthusiastische Unterstützung für den öffentlichen Rundfunk. Die Kritik an der Grösse und angeblichen Ineffizienz des öffentlichen Rundfunks hält an. Entsprechend hartnäckig halten sich auch Forderungen nach einer Verkleinerung dieser medialen Institution. 
Bei aller teilweise auch berechtigten Kritik an den Angeboten des öffentlichen Rundfunks und an den Leistungen privater Medien sollte aber der Blick aufs grosse Ganze nicht verloren gehen: Professionelle Informationsmedien leisten nach wie vor einen unverzichtbaren Beitrag für die Demokratie. Eine polemische Pauschalkritik an »den Mainstream-Medien" oder »den Staatsmedien « untergräbt das Vertrauen in Medien als zentrale Institution einer demokratischen Gesellschaft.

\section{Ausblick}

Unsere skizzierten Thesen legen nahe: Die mediale Agora wankt gewaltig. Aber es gibt Gegenkräfte, auch in der Schweiz, die diese Entwicklung zumindest hemmen können.

Erstens geniessen die professionellen Informationsmedien in vielen Ländern noch immer ein relativ hohes Vertrauen. Das Vertrauen in Medien ist in der Schweiz - nach den skandinavischen Ländern - noch immer relativ hoch (vgl. Reuters Institute, 2018). Besonders der öffentliche Rundfunk gilt als verlässlicher, vertrauenswürdiger Akteur. Auch die Qualität der Schweizer Medien ist bei vielen Titeln immer noch relativ hoch und die befragten Nutzer zeigen sich mit dem Angebot noch zufrieden (vgl. Medienqualität Schweiz, 2018). Klar ist aber auch, dass die Medienkonzentration zunimmt und die Medien unter immer grösserem Spardruck stehen. Beides wirkt sich messbar negativ auf die Qualität aus.

Zweitens hemmt die konkordanzorientierte politische Kultur der Schweiz die beschriebenen desintegrativen und desinformierenden Kräfte. So lehrt uns die Forschung, dass Desinformation vor allem in polarisierten Gesellschaften einen günstigen Nährboden findet. Entsprechend ist eine den Kompromiss fördernde politische Kultur, wie sie in der Schweiz noch existiert, ein wichtiger Puffer. Ebenso wichtig sind Medienkompetenz und die politische Bildung. Denn wer um die Rolle und Funktionsweise von Medien und politischen Entscheidungen weiss, kann mehr Verständnis dafür entwickeln, wenn die Medienberichterstattung oder wenn ein politischer Entscheid einmal nicht dem entspricht, was man sich gewünscht hat.

Drittens sorgt die Kleinräumigkeit der Schweiz dafür, dass der Markt für kommerziell betriebene $>$ Fake news -Angebote schlicht zu klein ist. Während im US-amerikanischen Wahlkampf Betreibende von Websites mittels Falschmeldungen Geld mit Werbung verdienen konnten, ist dies in der Schweiz kaum möglich. Gleichzeitig bedeutet die Kleinheit des Medienmarktes der Schweiz aber auch, dass ein aufwändiger Journalismus sich nicht vollständig am freien Markt refinanzieren kann.

Medienpolitisch gesehen stellt sich vor dem Hintergrund der beschriebenen Herausforderungen die Frage nach der Finanzierung der medialen 
Agora mit Nachdruck. Anstatt darauf zu vertrauen, dass journalistische Medienorganisationen trotz des enormen Einflusses der Tech-Giganten die digitale Transformation aus eigener Kraft schaffen, ist es an der Zeit, den Journalismus deutlich aktiver zu unterstützen. Eine direkte öffentliche Finanzierung respektive Förderung auch von privaten Onlinemedien scheint uns unabdingbar und sinnvoll, wenn die Vergabe an klare Bedingungen geknüpft ist (z. B. Investition in journalistische Ressourcen; Fokus auf Hintergrundinformationen etc.). Zudem sollte ernsthaft geprüft werden, Abgaben auf die Werbeeinnahmen von globalen Tech-Intermediären und Werbefenstern von deutschen und französischen Privatsendern zu erheben. Mit diesen Mitteln könnte ein Schweizer Fonds zur Förderung des Informationsjournalismus ins Leben gerufen werden.

Journalismus darf nicht auf ein Minimum reduziert, sondern muss gestützt und gestärkt werden. In einer zunehmend komplexen Welt braucht es starke Informationsmedien, die genau diese Komplexität auf kluge Weise reduzieren und vermitteln können. Und deshalb sind kluge Netzwerke aus Medien, Politik und Zivilgesellschaft gefragt, die kluge Ideen für eine nachhaltige Ausgestaltung eines sowohl privat wie öffentlich finanzierten Mediensystems einbringen.

\section{Literatur}

Arendt, Hannah (1992): Vita activa oder vom tätigen Leben. München Zürich: Piper.

Eisenegger, Mark (2017): Digitaler Strukturwandel der Öffentlichkeit - professionelle Informationsmedien nötiger denn je. In: fög - Forschungsinstitut Öffentlichkeit und Gesellschaft / Universität Zürich (Hg.): Jahrbuch Qualität der Medien - Schweiz Suisse Svizzera. Basel: Schwabe, 7-16.

eMarketer Chart (2017): Net US Digital Ad Revenues, by Company, 2016-2019 (billions). Abgerufen unter: https://www.emarketer.com/Chart/Net-US-Digital-Ad-Revenues-byCompany-2016-2019-billions/211332 (Stand: 28.12.2018).

fög - Forschungsinstitut Öffentlichkeit und Gesellschaft / Universität Zürich (Hg.) (2018): Jahrbuch Qualität der Medien - Schweiz Suisse Svizzera. Basel: Schwabe. Abgerufen unter: https://www.qualitaet-der-medien.ch/downloads (Stand: 28.12.2018)

Imhof, Kurt (1998): Digitale Agora? Das Internet und die Demokratie. In: Schweizerische UNESCO-Kommission (Hg.): INTERNET'97 - Etat des lieux et perspective. Bern: Swiss Commission for UNESCO, 121-130.

Kalogeropoulos, Antonis; Newman, Nic (2017): »I Saw the News on Facebook« - Brand Attribution when Accessing News from Distributed Environments. Digital News Project. Oxford: Reuters Institute for the Study of Journalism. Abgerufen unter: https://reutersins titute.politics.ox.ac.uk/sites/default/files/2017-07/Brand\%20attributions\%20report.pdf (Stand: 28.12.2018)

Lucht, Jens; Udris, Linards; Vogler, Daniel (2018): Politische Inszenierungen. Eine Inhaltsund Resonanzanalyse der Facebook-Seiten bundesdeutscher Parteien. Durchgeführt vom Forschungsinstitut Öffentlichkeit und Gesellschaft der Universität Zürich (fög) im Auftrag der Friedrich-Ebert-Stiftung. Bonn: Friedrich-Ebert-Stiftung. Abgerufen unter: http://library.fes.de/pdf-files/akademie/14035.pdf (Stand: 28.12.2018) 
Marconi, Francesco (2015): The rise of 'homeless` media. Abgerufen unter: https://me dium.com/thoughts-on-media/the-rise-of-homeless-media-97e031c8b319 (Stand: 28.12. 2018).

Medienqualität Schweiz (2018): MQR 18 Medienqualitätsrating 2018. Abgerufen unter: www.mqr-schweiz.ch (Stand: 28.12.2018)

Rauchfleisch, Adrian; Vogler, Daniel; Eisenegger, Mark (2017): Auf zu neuen Märkten: Der Einfluss Schweizer Medien auf Twitter. Zürich: fög - Forschungsinstitut Öffentlichkeit und Gesellschaft. Abgerufen unter: https://www.foeg.uzh.ch/dam/jcr:e4152c8d-a470 -4df0-804d-61e6f13aced5/Twitter-Analyse_3.pdf (Stand: 28.12.2018

Reuters Institute for the Study of Journalism (2018): Digital News Report 2018. Oxford: University of Oxford. Abgerufen unter: http://media.digitalnewsreport.org/wp-content/ uploads/2018/06/digital-news-report-2018.pdf?x89475 (Stand: 28.12.2018)

Schneider, Jörg; Eisenegger, Mark (2016): Wie Mediennutzer in die Welt schauen. Die Newsrepertoires der Schweizerinnen und Schweizer und ihre Themenagenden. In: Studien Qualität der Medien, Nr. 2/2016. Abgerufen unter: https://www.qualitaet-der-medien. ch/studienqdm (Stand: 28.12.2018)

Schneider, Jörg; Eisenegger, Mark (2018): Newsrepertoires junger Erwachsener. In: Nicole Gonser (Hg.): Der öffentliche (Mehr-)Wert von Medien: Public Value aus Publikumssicht. Wiesbaden: Springer Fachmedien, 93-107.

Schulz, Anne (2018): Where populist citizens get the news. An investigation of news audience polarization along populist attitudes in 11 countries. In: Communication Monographs 67, 1-24. Abgerufen unter: https://www.tandfonline.com/doi/full/10.1080/03637 751.2018.1508876 (Stand: 28.12.2018)

Schweizerische Eidgenossenschaft (2018): Vernehmlassung: Entwurf eines neuen Bundesgesetzes über elektronische Medien. Erläuternder Bericht. Bern.

Stiftung Werbestatistik Schweiz (2018): Werbeaufwand 2018. Abgerufen unter: https:// werbestatistik.ch/de/downloads/publikation-2018-1/summary.pdf-1 (Stand: 28.12.2018). Stieglitz, Stefan; Dang-Xuan, Linh (2012): Impact and diffusion of sentiment in public communication on Facebook. In: ECIS 2012, Nr. 98. Abgerufen unter: https://pdfs.semanti cscholar.org/bfac/3dde26f10e9e2a04a1813a6ee79c7f2b98e5.pdf (Stand: 28.12.2018)

Stieglitz, Stefan; Dang-Xuan, Linh (2013): Emotions and Information Diffusion in Social Media-Sentiment of Microblogs and Sharing Behavior. In: Journal of Management Information Systems 29, Nr. 4, 217-248. Abgerufen unter: https://www.tandfonline.com/doi/ abs/10.2753/MIS0742-1222290408 (Stand: 28.12.2018)

von Matt, Othmar (2018): Die unheimliche Macht der US-Giganten: Google und Facebook pflügen den Schweizer Medienmarkt um. In: Schweiz am Wochenende, 23.6.2018. Abgerufen unter: https://www.aargauerzeitung.ch/schweiz/die-unheimliche-macht-der-usgiganten-google-und-facebook-pfluegen-den-schweizer-medienmarkt-um-132724087 (Stand: 28.12.2018)

Wessler, Hartmut (2018): Habermas and the media. Cambridge: Polity Press. 